\section{EMBRYAIDDLE \\ Aeronautical University}

SCHOLARLY COMMONS

\section{International Journal of Aviation,} Aeronautics, and Aerospace

\title{
Real-Time Urban Weather Observations for Urban Air Mobility
}

\author{
Kevin A. Adkins \\ Embry-Riddle Aeronautical University, adkinsk@erau.edu \\ Mustafa Akbas \\ akbasm@erau.edu \\ Marc Compere \\ Embry-Riddle Aeronautical University - Daytona Beach, comperem@erau.edu
}

Follow this and additional works at: https://commons.erau.edu/ijaaa

Part of the Aerospace Engineering Commons, Aviation Commons, Computer Engineering Commons, Computer Sciences Commons, Earth Sciences Commons, Electrical and Computer Engineering Commons, and the Environmental Sciences Commons

\section{Scholarly Commons Citation}

Adkins, K. A., Akbas, M., \& Compere, M. (2020). Real-Time Urban Weather Observations for Urban Air Mobility. International Journal of Aviation, Aeronautics, and Aerospace, 7(4). https://doi.org/10.15394/ ijaaa.2020.1540

This Article is brought to you for free and open access by the Journals at Scholarly Commons. It has been accepted for inclusion in International Journal of Aviation, Aeronautics, and Aerospace by an authorized administrator of Scholarly Commons. For more information, please contact commons@erau.edu. 
It is estimated that more than $55 \%$ of the world's population live in urban areas today; this proportion is expected to jump to almost $70 \%$ by 2050 (United Nations, 2019). Presently, in North America, the population in urban areas is already estimated at $82 \%$ (United Nations, 2019). As the size of the urban population grows, the urban environment will be increasingly tasked to sustain the activities of a larger portion of the world's population. The urban boundary layer (UBL), the portion of the atmospheric boundary layer (ABL) whose climatic characteristics are modified by the presence of a city (Oke, 1976), is this environment. However, the UBL is one of the most complex and least understood microclimates due to the heterogeneity of the fabric underlying it and roughness elements composing it, omnipresent turbulence and anthropogenic heat sources, among other complex processes.

Cities of the future will have to overcome congestion, air pollution and increasing infrastructure cost while moving more people and goods smoothly, efficiently and in an eco-friendly manner. Urban air mobility (UAM), defined as a system that enables on-demand, highly automated, passenger- or cargo-carrying air transportation services (Lascara et al., 2019), is expected to be an integral component of achieving this new type of city (Xu, 2020). According to a Morgan Stanley research study, the market for autonomous urban air vehicles is expected to approach $\$ 1.5$ trillion globally by 2040 (Morgan Stanley, 2018). This same study also identified more than 240 UAM vehicles currently in development. These development efforts include all of the major aviation manufacturers and substantial investments by many traditional automotive companies, along with several innovative and well-funded startup companies. Further, even at this nascent stage, there are over 50 cities around the world pioneering urban air mobility programs (Unmanned Airspace, 2018a, 2018b). In the United States, the Federal Aviation Administration (FAA) has issued Part 135 U.S. air carrier certification to Wing Aviation LLC, a subsidiary of Google's parent corporation Alphabet. This was quickly followed by a similar issuance to UPS Flight Forward, Inc. More recently, Amazon Prime Air also achieved this certification. The FAA has indicated that five additional Part 135 air carrier certificates are under review at the time of this writing (Federal Aviation Administration, 2019).

Most prospective UAM operators envision a scenario of hundreds or thousands of simultaneous flight operations occurring over a large metropolitan area within an altitude block stretching from the surface to 5,000 feet, with nominal cruising altitudes of 1,000 - 2,000 feet above ground level (AGL) (Embraer X, 2020; Lascara et al., 2019; Porsche Consulting, 2018; Uber, 2016; Xu, 2020). This vision places these high-tempo flight operations squarely in the UBL. While traditional manned aircraft have quickly passed through this domain during ascent and descent, this is a new environment for sustained aviation operations. The heterogeneity of the urban fabric underlying the UBL and the roughness elements 
within it create a unique environment with very strong gradients. Consequently, flight conditions can change frequently and dramatically across very short temporal and spatial scales. Unfortunately, the lower mass and moment of inertia, and limited thrust and speed of UAM vehicles increase their sensitivity to these ambient atmospheric conditions. Therefore, the combination of these conditions and these aircraft susceptibilities necessitate meteorologically resolving the urban environment at small scales.

UAM vehicles face unique challenges related to weather in an urban operating environment. Without good weather products, aircraft may be unnecessarily grounded, delaying the delivery of goods and services and decreasing profitability. Regrettably, the staple aviation weather products for observations and forecasts at an airport on the outskirts of a metropolitan area do not translate well to the heterogeneous UBL and do not offer an appropriate spatial or temporal resolution for urban flight operations (Chapman, 2015; Meir et al., 2017; Napoly et al., 2018). Consequently, appropriate weather data for low-altitude urban airspace is needed and will be particularly critical for unlocking the full potential of UAM. Meteorological hazards of concern include wind and turbulence, temperature, ceiling and visibility, precipitation and icing. In addition to ensuring the safety and comfort of passenger-carrying aerial vehicles and the performance of cargo delivery aircraft, reliable weather information will be required for flight planning, flight authorizations, real-time adaptive trajectory planning and managing dynamic airspace boundaries, along with contingency planning.

Dense atmospheric observational networks sufficient for resolving the inhomogeneity of the UBL using traditional meteorological sensors are most likely impractical due to initial installation and continuing maintenance costs. The National Research Council of the National Academies (Committee on Urban Meteorology, 2012) highlights the great potential in using non-traditional sensor networks for observations of the urban atmosphere. Advances in the miniaturization of electronics, batteries, communications and cloud-based storage, along with a reduction in the cost of electronics and IoT sensors, provides an unprecedented opportunity to create high resolution observational networks that deliver real-time data that can complement traditional sources of meteorological observations (David 2019).

Dense urban meteorological observation networks comprised of crowdsourced data will enable a more complete picture of actual urban weather conditions required by UAM. This is especially the case in urban locales where routine weather observations are typically scarce but 'human sensors' and sensors associated with urban infrastructure are prevalent and dense. Besides supporting now-casting, dense urban meteorological observation networks comprised of crowdsourced data have the means to provide data for numerical weather prediction (NWP) data assimilation and validation of micro-scale forecasts (Agüera-Pérez et 
al., 2014; NCEP Central Operations, 2018; Overeem et al., 2013b; Ronda et al., 2017). Crowdsourcing can accomplish this in an effective, decentralized, economical and prompt manner (Frigerio et al., 2018). Hence, crowdsourced meteorological observation networks empower UAM by providing much needed data and a new class of essential weather products.

Section two of this paper suggests potential observation sensors and sources. Section three builds on this and puts forth an overarching architecture for the complete cyber-physical system. The proposed architecture includes an incentive-based crowdsensing application.

\section{Crowdsourced Weather Data}

\section{Crowdsourcing for Weather}

The idea of crowdsourcing was first introduced by Howe, who defined it as, "the act of taking a job traditionally performed by a designated agent (usually an employee) and outsourcing it to an undefined, generally large group of people in the form of an open call" (Howe, 2006). As the general idea of crowdsourcing has evolved, it is now understood to entail more than simply outsourcing a task, in this case the collection of data, but also the storage, quality control and utilization of the data (Hintz et al., 2019). However, the meteorological community itself has not yet reached broad consensus with respect to crowdsourcing nomenclature. The United Kingdom's Met Office suggests terminology that differentiates crowdsourced data and citizen-science data under the following structure (Hintz et al., 2019):

a) Citizen-science data: Data that is obtained from invited participants

b) Crowdsourced data: Data obtained from a group of people without explicit involvement

c) Opportunistic data: Data obtained from non-meteorological sensors

d) Third-party data: Data collected from meteorological sensors by a third-party

In contrast, the European Centre for Medium-Range Weather Forecasts (ECMWF) puts forth four categories within the crowdsourced moniker (Hintz et al., 2019):

a) Private and third party

b) Automated amateur weather stations

c) Smart connected devices

d) Human reporting

Evident in the contrasting of these two structures is the disparity between the Met Office, which distinguishes between citizen-science, crowdsourced data, and third-party data and the ECMWF, that uses the term in a more comprehensive manner. In keeping with the latter usage, in this paper, crowdsourced data will be used as a collective term and examples are presented for each of the data categories. 
A thorough review of early atmospheric science crowdsourcing efforts prior to 2014 is provided by Muller et al (2015).

In addition to the evolution of how crowdsourcing is thought about, the sources and availability of data have also evolved. Much of this evolution has been brought about by the proliferation of the Internet of Things (IoT) and smart devices. IoT refers to a network of Internet-connected objects and provides any-place, anytime connectivity and data exchange (Ashton, 2009; Evans, 2011; Smith, 2012). As technological infrastructure has grown, IoT data has grown in its availability, granularity, and coverage (David, 2019; Warren et al., 2016).

Although the use of crowdsourcing is more prevalent in other disciplines, such as ecology (Dickenson et al., 2010) and astronomy (Kärnfelt, 2014), and while the atmospheric science community has been slower to adopt it, examples of utilization for environmental sensing do exist. The nature of this data can generally be categorized as coming from two types of sources: objective and subjective. The former type of data represents measured data collected from the surrounding physical world. The second data source type, subjective data, is often referred to as social weather data and is gathered from public opinions, reactions, feelings and feedback. Zhu et al. provides a broad review of crowdsourced social weather data and meteorological services (Zhu et al., 2019).

\section{Measured (objective) Weather Data}

Objective crowdsourced weather data can be generated by fusing professional weather observations, both public and private, along with professional-grade observations from weather-sensitive organizations in industry. However, crowdsourcing also enables the collection of measured data from objective sources that are not necessarily professional-grade weather stations. Consideration of this measurement strategy lowers the cost of data acquisition and increases the coverage and spatial resolution of observations. This data is especially beneficial when accompanied by geotemporal tags.

A smartphone has a number of embedded sensors conducive to providing meteorological insight and it is projected that there are more than six billion smartphones carried by the public worldwide (Price et al., 2018). Potential weather parameters that could be gathered from smartphones include temperature, pressure, relative humidity, and light intensity and each of these observations can easily be tagged with a high-precision geotemporal tag (Kim et al., 2016; Overeem et al., 2013b; Sosko \& Dalyot, 2017). Further, accelerometers embedded in smartphones have the ability to detect continuous motion that could signify the phone's movement outdoors, thus improving the validity of the environmental sensor (Niforatos et al., 2017).

Temperature sensors are placed in phones to prevent damage during battery recharging. These battery temperature readings have been correlated to ambient temperature with a straightforward heat transfer model to estimate urban canopy 
layer temperatures across a wide range of climate zones and, within each, a variety of urban microclimates (Overeem et al., 2013b). Chau (2019) improved upon this correlation using two linear regression models for phones found in and out of pocket. Over 10 million such readings over the course of two years were compared to sparsely spaced World Meteorological Organization (WMO) and private weather stations by Droste et al. (2017) who showed that distinct local climate zones (LCZs) can be identified using this strategy. Sosko and Dalyot (2017) extended the promise of modern mobile devices to provide authoritative weather data to include, in addition to temperature, relative humidity.

Smartphones come equipped with relatively accurate pressure sensors in order to assist with improved navigation inside buildings and altimetry. Smartphone pressure measurements are plagued by fewer observational issues than those associated with temperature and relative humidity because such measurements are valid both within and outside of structures and are not impacted by the underlying UBL surface fabric (Mass \& Madaus, 2014). Kim et al. (2015, 2016) showed that pressure readings obtained by smartphones can be on par with observations from South Korea's national automatic weather station network. While a number of investigators have shown the utility of pressure observations from smartphones (Hintz et al., 2019; McNicholas \& Mass, 2018; Price et al., 2018), Madaus et al. (2014, 2017) has demonstrated how exploiting such observations from loosely-regulated networks can improve the forecasting and analysis of short-term, high-impact weather events.

Mobility potentially improves the efficiency of a sensor and most modern vehicles have atmospheric pressure and temperature sensors for use in engine management computers. When compared to research grade weather stations, while pressure observations were less well matched, temperature measurements have shown consistent agreement across a variety of makes and models of vehicles (Anderson et al., 2012). In addition to direct sensors, ambient conditions can be gleaned from headlight status, fog light setting, antilock brake system (ABS) activation, braking intensity, along with vehicle velocity and acceleration values (Mahoney \& O’Sullivan, 2013). Rabiei et al. (2013) demonstrated a direct link between the speed of wiper blades and rainfall intensity. The utilization of ground vehicles equipped with weather sensors, along with smartphone front view video (Aihara et al., 2016), has already been proposed to reduce the impact of adverse weather on surface transportation systems (Dey et al., 2015; Mahoney \& O'Sullivan, 2013). It is presumed that as surface transportation systems continue to evolve and become more automated, in the same manner as UAM systems, that the number of environmental sensors on vehicles, both surface and aerial, will continue to increase and be available for incorporation into a meteorological network.

The more substantive infrastructure associated with urban areas, especially in burgeoning smart cities, provide unique opportunities for the creation of dense 
observational networks. Dense networks, even when composed of a large set of basic stations with inferior performance, have been shown to outperform a smaller number of high-quality data stations (Agüera-Pérez et al., 2014). Infrastructure associated with air pollution monitoring is already intended for environmental monitoring and is a ready candidate for the enhancement of any broader meteorological observation network (Kumar et al., 2015; Moltchanov et al., 2015). However, infrastructure whose primary purpose is not associated with environmental monitoring, such as surveillance cameras, can also be employed. For example, surveillance cameras, that are prevalent across urban areas, have been shown to be effective for monitoring visibility (Wong et al., 2007, 2009).

Commercial microwave links (CMLs) transmit and receive microwave signals through the atmosphere and are widely deployed in telecommunications networks. Atmospheric conditions and weather phenomenon interfere with the propagation of these electromagnetic waves and hence CMLs can serve as a valuable real-time weather forecasting tool (Alpert et al., 2016; David \& Gao, 2017; Gosset et al., 2016; Overeem et al., 2013a). Messer et al. (2006) first demonstrated that CML signal attenuation can be used to estimate rainfall and subsequent investigations have continued to develop this potential (Berne \& Uijlenhoet, 2007; Chwala et al., 2012; Fencl et al., 2015; Leijnse, 2007a/2007b; Overeem et al., 2011; Zinevich et al., 2010). When CMLs are brought together into a network, high spatial and temporal precipitation maps can be developed that are superior to those commonly provided by standard weather services (Biachi et al., 2013; Fencl et al., 2017; Haese et al., 2017; Liberman et al., 2014; Sendik \& Messer, 2015; Troemel et al., 2014;Uijenhoet et al., 2018; Zinevich et al., 2008). In addition to identifying temperature inversions (David \& Gao, 2016), propagation delays between CMLs caused by atmospheric moisture have also been used to detect and monitor fog (Chwala et al., 2014; David, 2018; David \& Gao, 2018; David et al., 2009; Kawamura er at., 2017).

A growing number of individuals own personal weather stations (PWS; Bell et al., 2013) and the quantity of PWSs tend to be greater in heavily populated locales, such as cities (Vos et al., 2018). An increasing number of these weather stations are enabled to share real-time measurements with online platforms (Gharesifard \& When, 2016). Examples of online platforms include Weather Underground (wunderground.com), the Weather Observation Website (www.netoffice.gov.uk), and the Citizen Weather Observer Program (xwqa.com). PWS data has been employed successfully in a number of urban investigations. Temperature observations have been garnered from PWSs for Urban Heat Island investigations (Chapman et al., 2017; Meier et al., 2017; Steeneveld et al., 2011; Wolters \& Bransma, 2012). Intra and inter LCZ variability of air temperature was investigated using PWS data by Fenner et al. (2017). PWS acquired rainfall data has exhibited a superior resolution and quality of measurement than real-time 
unadjusted radar products (Vos et al., 2017). The incorporation of PWS data, amongst other unconventional data, has also been shown to improve the forecasting of convection (Gasperoni et al., 2018; Madaus et al., 2014; Sobash \& Stensrud, 2015).

Efforts to achieve high resolution spatial and temporal observations for UAM must necessarily include consideration of the vertical dimension. UAM vehicles in flight make for an excellent sensor platform and will be readily available for making observations aloft. Combining aerial vehicle hosted sensors with surface-based IoT observational networks (Chapman \& Bell, 2018) will allow for the realization of true three-dimensional observational networks. The use of crowdsourced air traffic control data for upper air meteorological monitoring has been demonstrated (Trub et al., 2018). Trub et al. inferred high quality wind velocity, temperature and pressure data, suitable for assimilation into numerical weather models, from continuously transmitted Secondary Surveillance Radar Mode S and ADS-B transponder signals crowdsourced from over 15 billion transponder messages. In addition, unmanned aerial vehicles themselves have been shown to be effective in persistent IoT monitoring of the near-surface environment (Cheng et al., 2013; Hernández-Vega et al., 2018; Rossi et al., 2018).

\section{Social (subjective) Weather Data}

Social weather data can be acquired via deliberate observation or inadvertently during daily life (Zhu t al., 2019). The multimodal nature of social weather data creates a rich tapestry of heterogeneous data stemming from texts, images, opinions, and behavioral information. Rossi et al. shows that people's attitude toward meteorological phenomenon can be mined from social networks (Rossi et al., 2018). Surface transportation research has used crowdsourced data to model and predict traffic speed by extracting weather events from Twitter (Lin et al., 2015) and $\mathrm{Lu}$ et al. (2018) extracted temporal, spatial, and meteorological features from social media to create a city-level traffic awareness alerting model that negates the need to fuse data from physical sensors operated by disparate government entities. Morita et al. (2018) developed a system that can collect, analyze and deem the location and severity of a disaster in real time based on information posted to social media. Geo-tagged pictures from web images have also been mined to extract snow-related information in inaccessible or mountainous regions (Guiliani et al., 2016).

In addition to mining popular social networks, an assortment of apps have been developed to more directly acquire social weather data. Social.Water is a software package developed for hydrological measurements that collects, parses, and categorizes text messages sent to a dedicated phone number Fienen \& Lowry, (2012). Niforatos et al. (2017) developed a crowdsourcing weather app that both samples smartphones' built-in sensors and allows for users to enter their own estimates of current and future weather conditions. The NoiseSpy (Kanjo, 2010), 
NoiseMap (Schweizer et al., 2012) and Ear-Phone (Rana et al., 2010) apps have enabled the mapping of sound levels to characterize urban noise pollution. The Mobile Application for Emergency Response and Support (MAppERS) enables those impacted by floodwaters to provide their location and status to authorities, along with snapshots that can be directly analyzed in support of the decisionmaking process during the crisis (Frigerio et al., 2018). Using a low-cost, massproducible iPhone add-on, the Spectropolarimeter for Planetary Exploration (SPEX) for iPhone (iSPEX), Snik et al. (2014) formed an atmospheric measurement network that permitted users to measure optical thickness to access the impact of aerosols on health, climate and air traffic. The AirTick mobile app has been developed to turn any smartphone camera into an air quality sensor that uses image analytics and deep learning techniques to supplement air quality monitoring (Pan et al., 2017). In the United States, the National Oceanic and Atmospheric Administration's (NOAA) Severe Storms Laboratory initiated the social crowdsourcing project, Meteorological Phenomena Identification Near the Ground (mPING). Any member of the public, without registration, can submit a weather report and the initiative has shown the capability to provide consistent and accurate observational data (Elmore et al., 2014). Crowdsourced social weather data fuses a large heterogeneous dataset. However, the associated subjective opinions and individual participation ultimately provides large-scale, high-density information (Zhu et al., 2019). The gathering of such data by fixed ground-based sensors deployed over the same geographical area would be cost-prohibitive and impractical (Strangeways, 2018).

\section{Real-Time Urban Observations for Aviation via Crowdsensing}

Pervasive deployment of IoT devices has enabled the implementation of a new form of crowdsourcing application: crowdsensing (Ganti et al., 2011). In crowdsensing, the sensed data from various stationary and mobile IoT sources, both of which would be present in a UAM application, are automatically but strategically connected. The crowdsensing participants, which can be both users and sensorequipped devices, are generally required to complete tasks according to an intentionally chosen strategy. Further, the manner in which the mobile sources in the crowdsourcing application participate must be deliberately specified.

Real-time urban meteorological observations, and in particular those for enabling UAM, can benefit greatly from advances in crowdsensing technologies. To the best of our knowledge, there is no crowdsensing solution designed for UAM weather forecasting and operations. However, solutions developed for other applications, with mobile elements present, provide valuable lessons. Using the key insights from these applications, we present a data collection framework and an incentive-based crowdsensing application for real-time urban observations in this section. The goal of the framework is to capture high-resolution spatial and 
temporal meteorological data for the creation, reporting and validation of weather forecasting products in support of UAM.

In the following subsections, we first present the overarching architecture of the cyber-physical system and then present the main components of the incentive-based crowdsensing application and list the important design considerations.

\section{System Architecture}

The common methodology used to describe the architecture of a networkbased solution is to detail the network layers and the corresponding services found in each of these layers. This section presents a four-layer architecture (Figure 1) similar to the architectural layers for mobile crowdsensing systems by Capponi et al. (2019).

\section{Figure 1}

Real-Time Urban Observation System Architecture.

\begin{tabular}{|c|l|l|}
\hline Application Layer & $\begin{array}{l}\text { - Incentive-Based Crowdsensing Application } \\
\text { Data Layer }\end{array}$ & $\begin{array}{l}\text { - Data Storage \& Management } \\
\text { - Initial Data Analysis }\end{array}$ \\
\hline Network and & $\begin{array}{l}\text { - Urban Communication Technologies } \\
\text { - Data Sensing Mode Control }\end{array}$ \\
\hline Communications Layer & $\begin{array}{l}\text { - Data Collection Network Elements } \\
\text { (IoT, UAVs, Smart Phones, Weather Stations, etc.) }\end{array}$ \\
\hline
\end{tabular}

\section{Physical Sensing Layer}

The physical sensing layer includes all of the data sensing elements that would be used in our solution as data collection participants. A data collector can be a single IoT device such as a temperature sensor with a controller or an unmanned aerial vehicle with numerous onboard sensors. The network elements at this layer will form the endpoints of our data collection network and sense data according to their capabilities and the sensing strategies. In networking terminology, the physical sensing layer includes only the devices and their physical communication, such as the wireless connection among sensors. The sensing strategies, such as periodic data collection, are implemented on the upper layers. Some examples of possible data collectors are: 
- Professional/Commercial Grade Weather Stations: Several weather sensitive industries site a variety of weather sensors. Further, several companies, such as Uber, have indicated that they plan on siting weather instrumentation at every vertiport and at select locations along common flight routes.

- Amateur/Personal Weather Stations: An increasing number of these weather stations are enabled to share real-time measurements online.

- Aerial Vehicles: Much like current traditional manned aircraft, urban aerial vehicles will be equipped with onboard weather sensing capabilities.

- IoT: All IoT devices with sensors that are connected to the urban network, especially in burgeoning smart cities. These can include: light sensors, temperature sensors, wind sensors, acoustic sensors, video cameras, etc.

- Ground Vehicles: Both traditional ground vehicles and burgeoning autonomous vehicles are equipped with numerous sensors that can be used for data collection in the urban network.

- Personal Devices: Human participants can choose to use their personal mobile devices or wearable devices for data collection by either recording their own observations manually or through an application running in the background that records and transmits sensor readings.

- Government meteorological infrastructure: This can include sensing elements for air pollution monitoring or transportation systems.

These network elements will each be used in different roles for data collection based on their properties in the system. For instance, while the typical role of a device is simply data collection, devices with suitable telecommunication capabilities can be used for both data collection and relay and in that serve as a central unit of a small data collection cluster. Additionally, while some of the devices are expected to be functional all of the time, such as stationary weather observation stations, others will be used dynamically based on the system requirements.

\section{Network and Communications Layer}

The network and communications layer includes the technology that provides connectivity among the disparate network elements in the physical sensing layer. Hence, this layer is the glue that holds the entirety of the system together. All existing and expected urban telecommunication technologies are utilized in this layer. These include both wireless (Wi-Fi, LTE, 5G, Bluetooth, etc.) and wired (Cable, DSL, FTTH, etc.) technologies. Advantages and disadvantages include:

- Wi-Fi: Wi-Fi is the most common form of wireless technology used by urban crowdsensing participants. It also has a cost advantage compared to most other wireless technologies. However, its main disadvantage is its limited mobile connectivity. Therefore, Wi-Fi can be considered as a location-based option for the data collection in our application. 
- LTE: Cellular connectivity is one of the critical components of any mobile crowdsensing solution in an urban environment. Both the users of mobile devices and the aerial vehicles leverage the opportunities provided by cellular connectivity. Current $4 \mathrm{G}$ and LTE solutions provide reasonably good data rate and delay performance for data collection.

- 5G: $5 \mathrm{G}$ is expected to provide longer transmission ranges and support software defined networking applications. Both of these features will extend the capabilities of crowdsensing applications in an urban environment.

- Bluetooth: Bluetooth is a potentially good candidate for limited distance, energy-efficient data collection, particularly to dump data to a data collection point when other options are not available.

- Other wireless technologies: There are wireless technologies that use different frequencies other than the traditional options. For example, optical lasers or millimeter wave wireless communications have started to be used in several urban applications for data transmission.

- Wired networks: A multitude of various data collection sensors are anticipated to be incorporated into future smart cities. Additionally, data collected by wireless networks will be transferred to cloud data centers mostly by using the wired infrastructure.

In addition to the abovementioned technologies, the proposed system architecture allows incorporation of ad-hoc networking solutions as well. For instance, an unmanned aircraft (UA) swarm or a surface autonomous vehicle (AV) platoon can be disconnected from the overall system for some time and then dump timestamped data to the network when they reconnect.

The network and communications layer must offer network and sensing configuration capabilities. For example, an important consideration for real-time urban meteorological observations is the execution time of the sensing activity. One possible implementation strategy is synchronous sensing, where the sensed data is transmitted to the corresponding data center in real-time. This is in contrast to asynchronous sensing where the collected data is time-stamped and later transmitted according to a predefined plan. While synchronous sensing may seem to be ideal, it may require a large investment in terms of connectivity and communication technologies. Consequently, the selection of synchronous or asynchronous sensing must take into account the meteorological observation network's sensitivity to real-time requirements.

\section{Data Layer}

The data layer undertakes the storage, management and initial analysis of the vast amount of data collected by the observation system. The data collected by the network of sensing elements must be stored and processed to be used by the crowdsensing applications. For the storage and management of the data, there are database solutions, which can be implemented by using a central server or a 
distributed solution making use of recent edge computing solutions. The traditional crowdsensing approaches generally use a single location for data processing as it provides options to process data using large computational resources. However, some applications have less strict requirements for data preprocessing. Additionally, recent advances in edge computing provide more powerful distributed solutions. In our framework, we envision a single data processing location with possible extension to a distributed solution. The single location will provide a central control over the data collection strategy in the first stages of the design.

The data layer also includes the management and initial analysis of the collected data. The initial data quality check is done in this layer to remove noise. The data check and filtering allow the crowdsensing application to receive only filtered, high quality data. Additionally, machine learning-based methods can be embedded in the data layer to both detect anomalies and make predictions by using the real-time data flow.

\section{Application Layer}

The application layer is where all of the crowdsensing mechanisms are implemented and executed. The implementation includes the overall crowdsensing strategy and the network element configuration.

The crowdsensing strategy will be implemented by considering two types of users (or autonomous agents), data contributors and data collectors. The main challenges of crowdsensing applications are the recruitment of the contributors, selection among these and task assignment according to a certain crowdsensing campaign. Addressing the details of these aspects for our proposed application is given in the following section.

\section{Incentive-Based Crowdsensing Application}

Incentive mechanisms play an important role in crowdsensing solutions. These mechanisms use different forms of incentives or rewards to increase the number of participants and select participants based on the requirements of the applications (Ota et al., 2018; Sun, 2013; Zhang et al., 2016). The UAM meteorological application that we consider in this paper requires a large number of participants for fine-grained data. Additionally, the selection of these participants for data collection depends on their location at the intended data collection time.

The participants in the meteorological application can be various weather sensors, devices with embedded sensors, along with human sensors. If these sensors participate in the meteorological data collection, it will be possible to identify finegrained weather data. However, the data collectors would need a motive to participate in the crowdsensing system since it may also entail some minor disadvantages. For instance, if the data collection is an additional task for the sensor's regular sensing plan, it will require extra power consumption for 
observation and data transmission. Hence, a well-designed incentive mechanism will be of significant benefit for this meteorological application.

\section{Crowdsensing Application}

The main components of our incentive-based crowdsensing application are as follows:

- Task advertisement: The data collection tasks will be advertised to all potential participants. These tasks will have an incentive associated with them, which can be static or dynamic based on the needs of the system at the time.

- Participant selection: The participant selection for a specific task will depend on the current state of the system and the nature of the task. For instance, the spatial coverage, the mobility of the participant and the variability of its collected data will influence the selection.

- Task assignment: Once the participants are selected for task execution, the system will assign tasks with certain incentive values.

- Task execution: The participants for task execution can be the users of mobile devices or smart agents running on various devices or vehicles. Our application will allow both of these task execution types.

- Incentivizing: The participants will be rewarded as they execute tasks. Continually, the incentives and reputations of the users will be updated according to the state of the system.

The incentive-based crowdsensing application uses advertisements to recruit users or devices. An important parameter for the incentive mechanism is the form of the incentives. Since fine resolution environmental insight is invaluable for various smart city applications, a good example of an incentive is a discount to a subscription service that brings together weather data from a number of participants. In this scheme, participants would become members of a system from which they get service from and would do so at a discounted price. Simultaneously, they would also improve the same service as they collect and share data.

Following the recruitment of participants, the application selects users among the participants based on the particular goal at that point in time. The system assigns tasks to the selected participants and their data is contributed to the system. The collected data is invaluable since, in addition to ensuring the safety and comfort of passenger-carrying aerial vehicles and the performance of cargo delivery aircraft, it will be required for flight planning, flight authorizations, real-time adaptive trajectory planning and managing dynamic airspace boundaries, along with contingency planning.

\section{Incentivizing Strategy}

The strategy for the distribution of incentives is critical for the proposed crowdsensing application since it will play an important role in the recruitment and selection of participants. There are two main methods of distributing the incentives in crowdsensing applications. In dynamic incentive distribution strategies, the 
incentives are updated during the operation (Lou et al., 2014; Yang et al., 2012). Alternatively, static incentive distribution strategies distribute incentives offline before the operation and do not update them during the course of the operation (Tsujimori et al., 2013). Since the spatial and temporal requirements for meteorological data collection may change frequently, a dynamic strategy is more appropriate for this application. With this dynamic strategy, there are two important requirements that are central to its design:

- Data type, quality and quantity: The data of greatest interest in our application is data that addresses wind, turbulence, temperature, ceiling, visibility, precipitation and icing. Therefore, the tasks for the crowdsensing application will be the collection of these data types.

- Resource management: Participant recruitment and maintenance are fundamental challenges in collecting sufficient data for crowdsensing applications. While a higher number of participants are preferred for coverage, the cost associated with each participant must be taken into account.

These requirements shape the characteristics of the incentivizing strategy. Even though we would like to collect all available data for the most extensive spatial coverage, the application is unable to blindly accept every data point from all participants. Consequently, a reputation-based method must be incorporated into the strategy in order to assess the quality of the collected data by different participants. In addition, an optimization mechanism is required to keep a certain number of participants in the system for a given spatial coverage of the threedimensional urban environment, while staying in budget.

To satisfy the requirements of our crowdsensing application, we employ "Game Theory" when designing our incentive mechanism. Game Theory is a theoretical framework to conceive situations among competing players and produce optimal decision-making of independent and competing actors in a strategic setting (Ross, 2019). It is considered one of the most important analytical tools for studying systems, where a participant's best course of action for their own good is dependent upon their expectations about the other participants' actions. Therefore, it is an invaluable tool for crowdsensing systems with incentive mechanisms, particularly for applications with strategic interactions among multiple decision-makers (Osborne \& Rubinstein, 1994).

We use Game Theory to model the strategic interactions among the data collectors and the generation of an optimal configuration for the system's overall gain. This approach provides the theoretical framework for the strategy so that the dynamics of incentive advertisement and task assignment ends with a stable assignment for participants. The incentive mechanism is modeled as a "game" (per its definition in Game Theory) in which the participants are the players and the participants' trajectories, or their sensing times if they are stationary, are the strategies. In this game, we assume that the participants are individually rational in 
terms of data collection and act in their own interests. We would like to use these participants' data according to the system's spatial and temporal needs. Therefore, our system advertises incentives for executing sensing tasks and the value of a particular incentive is determined by the location and time. The participants decide whether to execute the sensing task or not based on the incentives. When a mobile participant is not satisfied with the current incentive at a location, it can check for other available nearby advertisements. If executing a sensing task in another location has a better incentive, then the participant can choose to move to that location. If the participant is stationary and the incentive is not satisfactory, it can choose to wait for another time.

\section{Implementation Plan}

The proposed network architecture, network elements and crowdsensing application, offer a real-time urban meteorological observation solution that can be used for granular meteorological data collection. Future work includes a plan to implement and evaluate the application in two phases. The first phase consists of setting up an experimental design containing a small number of different data collectors, such as smart phones and UAVs with hosted sensors. The primary goal of this phase is the proof-of-concept implementation that shows the roles of different network layers and the functioning of the data collection mechanism in a real-life setting.

Following the initial experiment, the application and the network architecture will be implemented in simulation, using realistic network communication and urban mobility components (Compere et al., 2019). The simulation will afford us the opportunity to test a multitude of what-if scenarios in an environment with a large number of crowdsensing participants. This is critical in order to evaluate the performance of the approach in a dense network. Additionally, we plan to implement a hybrid testbed-simulation approach to combine the real-life conditions of the testbed environment with the enhanced modeling capabilities of the simulation environment.

\section{Conclusion}

UAM is expected to be an integral component of future cities. However, this is a new environment for sustained flight operations. Without the availability of good weather products appropriate for this domain, safety will be jeopardized, thus decreasing public confidence, and the delivery of goods and services will be disrupted, thus decreasing profitability. The spatially and temporally dynamic environment of the UBL necessitates fine resolution meteorological observations. The requisite number of sensors to accomplish this makes a traditional observation network implausible in terms of installation and continuing maintenance costs. While crowdsensing offers numerous opportunities in the urban environment for the collection and real-time reporting of data, it has not yet been thoroughly 
explored for obtaining high-quality meteorological data for this purpose. In this paper, we introduce an overarching architecture for a cyber-physical urban meteorological observational system and an incentive-based crowdsensing application that is designed on top of this architecture for dynamic data collection in support of urban aviation operations.

\section{Funding Sources}

The authors declare no specific funding for this work. 


\section{References}

Agüera-Pérez, A., Palomares-Salas, J. C., de la Rosa, Juan José González, \& Sierra-Fernández, J. M. (2014). Regional wind monitoring system based on multiple sensor networks: A crowdsourcing preliminary test. Journal of Wind Engineering \& Industrial Aerodynamics, 127, 51-58. doi:10.1016/j.jweia.2014.02.006

Aihara K., Bin P., Imura H., Takasu A., \& Tanaka Y. (2016). On feasibility of crowdsourced mobile sensing for smarter city life. In N. Streitz \& P. Markopoulos (Eds.) Distributed, ambient and pervasive interactions. DAPI 2016. Lecture Notes in Computer Science, vol 9749. Springer.

Alpert, P., Messer, H., \& David, N. (2016). Meteorology: Mobile networks aid weather monitoring. Nature Publishing Group. doi:10.1038/537617e

Anderson, A., Chapman, M., Drobot, S., Tadesse, A., Lambi, B., Wiener, G., \& Pisano, P. (2012). Quality of mobile air temperature and atmospheric pressure observations from the 2010 development test environment experiment. Journal of Applied Meteorology and Climatology, 51, 691701. doi:10.1175/JAMC-D-11-0126.1

Ashton, K. (2009). That 'Internet of Things' thing: In the real world things matter more than ideas. RFID Journal. www.rfidjournal.com /articles/view?4986

Bell, S., Cornford, D., \& Bastin, L. (2013). The state of automated amateur weather observations. Weather, 68(2), 36-41. doi:10.1002/wea.1980

Berne, A., \& Uijlenhoet, R. (2007). Path-averaged rainfall estimation using microwave links: Uncertainty due to spatial rainfall variability American Geophysical Union. doi:10.1029/2007GL029409

Bianchi, B., Jan van Leeuwen, P., Hogan, R. J., \& Berne, A. (2013). A variational approach to retrieve rain rate by combining information from rain gauges, radars, and microwave links. Journal of Hydrometeorology, 14(6), 18971909. https://doi.org/10.1175/JHM-D-12-094.1

Capponi, A., Fiandrino, C., Kantarci, B., Foschini, L., Kliazovich, D., \& Bouvry, P. (2019). A survey on mobile crowdsensing systems: Challenges, solutions, and opportunities. IEEE Communications Surveys \& Tutorials, 21(3), 2419-2465. doi: 10.1109/COMST.2019.2914030

Chapman L. (2015). Urban meteorological networks: An urban climatologists panacea? Urban Climatology News, 58, 7-12. Retrieved from http://www.meteo.fr/icuc9/presentations/PLENARY/PLENARY3.pdf

Chapman, L., \& Bell, S. J. (2018). High-resolution monitoring of weather impacts on infrastructure networks using the internet of things. Bulletin of the American Meteorological Society, 99(6), 1147-1154. doi:10.1175/BAMSD-17-0214.1

Chapman, L., Bell, C., \& Bell, S. (2017). Can the crowdsourcing data paradigm take atmospheric science to a new level? A case study of the urban heat 
island of London quantified using Netatmo weather stations. International Journal of Climatology, 37(9), 3597-3605. doi:10.1002/joc.4940

Chau, N. H. (2019). Estimation of air temperature using smartphones in different contexts. Journal of Information and Telecommunication, 3(4), 494-507. doi:10.1080/24751839.2019.1634869

Cheng, Y., Li, X., Jia, J., Zhang, J., Lin, K., Liu, X., Li, Y., Jiang, X. (2013). An autonomous aerial system for air-quality surveillance and alarm. Journal of China Intel IoT Joint Labs, 13, 491-492.

Chwala, C., Gmeiner, A., Qiu, W., Hipp, S., Nienaber, D., Siart, U., . . . Kunstmann, H. (2012). Precipitation observation using microwave backhaul links in the alpine and pre-alpine region of southern Germany. Katlenburg-Lindau: Copernicus GmbH. doi:10.5194/hess-16-2647-2012

Chwala, C., Kunstmann, H., Hipp, S., \& Siart, U. (2014). A monostatic microwave transmission experiment for line integrated precipitation and humidity remote sensing. Atmospheric Research, 144, 57-72. doi:10.1016/j.atmosres.2013.05.014

Committee on Urban Meteorology: Scoping the Problem, Defining the Needs. (2012). Urban meteorology: Forecasting, monitoring, and meeting users' needs. National Academies Press. doi:10.17226/13328

Compere, M., Adkins, K., Legon, O., \& Currier, P. (2019). MoVE: A mobility virtual environment for testing multi-vehicle scenarios. Proceedings of 2019 NDIA Ground Vehicle Systems Engineering and Technology Symposium, Modeling \& Simulation, Testing and Validation (MSTV) Technical Session, August 13-15, 2019, Novi, Michigan. Retrieved from http://gvsets.ndia-mich.org/documents/MSTV/2019/MoVE_Mobility_ Virtual_Environment_Compere_Marc_12_June_2019.pdf

David, N. (2018). Utilizing microwave communication data for detecting fog where satellite retrievals are challenged. Natural Hazards, 94, 867-882. doi:10.1007/s11069-018-3428-3

David, N. (2019). Harnessing crowdsourced data and prevalent technologies for atmospheric research. Advances in Atmospheric Sciences, 36, 766-769.

David, N., \& Gao, H. O. (2016). Using cellular communication networks to detect air pollution. Environmental Science Technology, 50(17), 9442-9451. doi:10.1021/acs.est.6b00681

David, N., \& Gao, H. O. (2017). Atmospheric monitoring using commercial microwave networks. Proc. 15th International Conf. on Environmental Science and Technology, Rhodes, Greece, Global NEST, 1-4.

David, N., \& Gao, H. O. (2018). Using cell-phone tower signals for detecting the precursors of fog. Journal of Geophysical Research. doi:10.1002/2017JD027360 
David, N., Alpert, P., \& Messer, H. (2009). Technical note: Novel method for water vapour monitoring using wireless communication networks measurements. Atmospheric Chemistry and Physics, 9, 2413-2418. https://doi.org/10.5194/acp-9-2413-2009

Dey, K. C., Mishra, A., \& Chowdhury, M. (2015). Potential of intelligent transportation systems in mitigating adverse weather impacts on road mobility: A review IEEE. doi:10.1109/TITS.2014.2371455

Dickinson, J., Zuckerberg, B., \& Bonter, D. (2010). Citizen science as an ecological research tool: Challenges and benefits. Annual Review of Ecology, Evolution, and Systematics, 41(1), 149-172.

Droste, A. M., Pape, J. J., Overeem, A., Leijnse, H., Steeneveld, G. J., Van Delden, A. J., \& Uijlenhoet, R. (2017). Crowdsourcing urban air temperatures through smartphone battery temperatures in São Paulo, Brazil. Journal of Atmospheric and Oceanic Technology, 34(9), 18531866. doi:10.1175/JTECH-D-16-0150.1

Elmore, K. L., Flamig, Z. L., Lakshmanan, V., Kaney, B. T., Farmer, V., Reeves, H. D., \& Rothfusz, L. P. (2014). MPING: Crowd-sourcing weather reports for research. Bulletin of the American Meteorological Society, 95(9), 1335-1342. doi:10.1175/BAMS-D-13-00014.1

EmbraerX. (2020). Flight plan 2030: An air traffic management concept for urban air mobility. Retrieved from www.flightplan2030.com

Evans, D. (2011). The Internet of things: How the next evolution of the Internet is changing everything. Cisco Internet Business Solutions Group. www.cisco.com/web/about/ac79/docs/innov/IoT_IBSG_0411FINAL.pdf

Federal Aviation Administration. (2019). Package delivery by drone (Part 135). https://www.faa.gov/uas/advanced_operations/package_delivery_drone

Fencl, M., Dohnal, M., Rieckermann, J., \& Bares, V. (2017). Gauge-adjusted rainfall estimates from commercial microwave links. Katlenburg-Lindau: Copernicus GmbH. doi:10.5194/hess-21-617-2017

Fencl, M., Rieckermann, J., Sýkora, P., Stránský, D., \& Bareš, V. (2015). Commercial microwave links instead of rain gauges: Fiction or reality? IWA Publishing. doi:10.2166/wst.2014.466

Fenner, D., Meier, F., Bechtel, B., Otto, M., \& Scherer, D. (2017). Intra and inter 'local climate zone' variability of air temperature as observed by crowdsourced citizen weather stations in Berlin, Germany. Meteorologische Zeitschrift, 26, 525-547. doi:10.1127/metz/2017/0861

Fienen, M. N., \& Lowry, C. S. (2012). Social.Water-A crowdsourcing tool for environmental data acquisition. Computers and Geosciences, 49, 164-169. doi:10.1016/j.cageo.2012.06.015

Frigerio, S., Schenato, L., Bossi, G., Mantovani, M., Marcato, G., \& Pasuto, A. (2018). Hands-on experience of crowdsourcing for flood risks. an android 
mobile application tested in Frederikssund, Denmark. International Journal of Environmental Research and Public Health, 15(9), 1926. doi:10.3390/ijerph15091926

Ganti, R. K., Ye, F., \& H. Lei, H. (2011, November) Mobile crowdsensing: Current state and future challenges. IEEE Communications Magazine, 49(11), 32-39.

Gasperoni, N. A., Wang, X., Brewster, K. A., \& Carr, F. H. (2018). Assessing impacts of the high-frequency assimilation of surface observations for the forecast of convection initiation on 3 April 2014 within the Dallas-Fort Worth test bed. Monthly Weather Review, 146(11), 3845-3872. doi:10.1175/MWR-D-18-0177.1

Gharesifard, M., \& Wehn, U. (2016). To share or not to share: Drivers and barriers for sharing data via online amateur weather networks. Journal of Hydrology, 535, 181-190. doi:10.1016/j.jhydrol.2016.01.036

Giuliani, M., Castelletti, A., Fedorov, R., \& Fraternali, P. (2016). Using crowdsourced web content for informing water systems operations in snow-dominated catchments. Hydrology and Earth System Sciences, 20(12), 5049-5062. doi:10.5194/hess-20-5049-2016

Gosset, M., Kunstmann, H., Zougmore, F., Cazenave, F., Leijnse, H., Uijlenhoet, R., . . . Hoedjes, J. (2016). Improving rainfall measurement in gauge poor regions thanks to mobile telecommunication networks. American Meteorological Society. doi:10.1175/BAMS-D-15-00164.1

Haese, B., Hörning, S., Chwala, C., Bárdossy, A., Schalge, B., \& Kunstmann, H. (2017). Stochastic reconstruction and interpolation of precipitation fields using combined information of commercial microwave links and rain gauges. Water Resources Research, 53, 10,740-10,756. https://doi.org/10.1002/2017WR021015

Hernández-Vega, J. I., Varela E. R., Romero N. H., Hernández-Santos C., Cuevas J. L. S., \& Gorham, D. G. P. (2018). Internet of things (IoT) for monitoring air pollutants with an unmanned aerial vehicle (UAV) in a smart city. In F. Torres Guerrero, J. Lozoya-Santos, E. Gonzalez Mendivil, L. Neira-Tovar, P. Ramírez Flores, \& J. Martin-Gutierrez (Eds.) Smart Technology. Lecture Notes of the Institute for Computer Sciences, Social Informatics and Telecommunications Engineering, vol 213. Springer, Cham. https://doi.org/10.1007/978-3-319-73323-4_11

Hintz, K. S., O'Boyle, K., Dance, S. L., Al-Ali, S., Ansper, I., Blaauboer, D., . . . Yang, X. (2019). Collecting and utilizing crowdsourced data for numerical weather prediction: Propositions from the meeting held in Copenhagen, 45 December 2018. Atmospheric Science Letters, 20(7). https://doi.org/10.1002/asl.921 
Hintz, K. S., Vedel, H., \& Kaas, E. (2019). Collecting and processing of barometric data from smartphones for potential use in numerical weather prediction data assimilation. Meteorological Applications, 2019, 1-14. https://doi.org/10.1002/met.1805

Howe, J. (2006). Crowdsourcing: A definition. http://crowdsourcing.typepad.com/ cs/2006/06/crowdsourcing_a.html

Hu, Z., Bai, Z., Yang, Y., Zheng, Z., Bian, K., \& Song, L. (2019). UAV aided aerial-ground IoT for air quality sensing in smart city: Architecture, technologies, and implementation. IEEE Network, 33(2), 14-22. doi:10.1109/MNET.2019.1800214

Kanjo, E. (2010). NoiseSPY: A real-time mobile phone platform for urban noise monitoring and mapping. Mobile Networks and Applications, 15(4), 562574. doi:10.1007/s11036-009-0217-y

Kärnfelt, J. (2014). Knut lundmark, meteors and an early Swedish crowdsourcing experiment. Annals of Science, 71(4), 449-473. doi:10.1080/00033790.2013.827073

Kawamura, S., Ohta, H., Hanado, H., Yamamoto, M. K., Shiga, N., Kido, K., . . . Iguchi, T. (2017). Water vapor estimation using digital terrestrial broadcasting waves. doi:10.1002/2016RS006191

Kim, N., Kim, Y., Yoon, Y., Im, H., Choi, R. K. Y., \& Lee, Y. H. (2015). Correcting air-pressure data collected by MEMS sensors in smartphones. Hindawi. doi:10.1155/2015/245498

Kim, Y., Ha, J., Yoon, Y., Kim, N., Im, H., Sim, S., \& Choi, R. K. Y. (2016). Improved correction of atmospheric pressure data obtained by smartphones through machine learning. Computational Intelligence and Neuroscience, 9467878. doi:10.1155/2016/9467878

Kumar, P., Morawska, L., Martani, C., Biskos, G., Neophytou, M., Di Sabatino, S., . . Britter, R. (2015). The rise of low-cost sensing for managing air pollution in cities. Elsevier Ltd. doi:10.1016/j.envint.2014.11.019

Lascara, B., Lacher, A. R., DeGarmo, M. T., Maroney, D. R., Niles, F. A., \& Vempati, L. (2019). Urban air mobility airspace integration concepts: Operational concepts and exploration approaches -19-00667-9. Retrieved from https://www.mitre.org/publications/technical-papers/urban-airmobility-airspace-integration-concepts

Leijnse, H., Uijlenhoet, R., \& Stricker, J. N. M. (2007a). Hydrometeorological application of a microwave link: 2. Precipitation. doi:10.1029/2006WR004989

Leijnse, H., Uijlenhoet, R., \& Stricker, J. N. M. (2007b). Rainfall measurement using radio links from cellular communication networks American Geophysical Union. doi:10.1029/2006WR005631 
Liberman, Y., Samuels, R., Alpert, P., \& Messer, H. (2014). New algorithm for integration between wireless microwave sensor network and radar for improved rainfall measurement and mapping. Atmospheric Measurement Techniques, 7(10), 3549-3563.

Lin, L., Ni, M., He, Q., Gao, J., \& Sadek, A. W. (2015). Modeling the impacts of inclement weather on freeway traffic speed: Exploratory study with social media data. Transportation Research Record: Journal of the Transportation Research Board, 2482(1), 82-89. doi:10.3141/2482-11

Lu, H., Zhu, Y., Shi, K., Lv, Y., Shi, P., \& Niu, Z. (2018). Using adverse weather data in social media to assist with city-level traffic situation awareness and alerting. Applied Sciences, 8(7), 1193.

Luo, T., Tan, H. P., \& Xia, L. (2014, April). Profit-maximizing incentive for participatory sensing. In IEEE Conference on Computer Communications (INFOCOM), Apr 2014, 127-135.

Madaus, L. E., \& Mass, C. F. (2017). Evaluating smartphone pressure observations for mesoscale analyses and forecasts. American Meteorological Society. doi:10.1175/WAF-D-16-0135.1

Madaus, L. E., Hakim, G. J., \& Mass, C. F. (2014). Utility of dense pressure observations for improving mesoscale analyses and forecasts. American Meteorological Society. doi:10.1175/MWR-D-13-00269.1

Mahoney, W. P., \& O'Sullivan, J. M. (2013). Realizing the potential of vehiclebased observations. Bulletin of the American Meteorological Society, 94(7), 1007-1018.

Mass, C., \& Madaus, L. (2014). Surface pressure observations from smartphones: A potential revolution for high-resolution weather prediction? Bulletin of the American Meteorological Society, 95(9), 1343-1349. doi:10.1175/BAMS-D-13-00188.1

McNicholas, C., \& Mass, C. F. (2018). Smartphone pressure collection and bias correction using machine learning. American Meteorological Society. doi:10.1175/JTECH-D-17-0096.1

Meier, F., Fenner, D., Grassmann, T., Otto, M., \& Scherer, D. (2017). Crowdsourcing air temperature from citizen weather stations for urban climate research. Urban Climate, 19, 170-191. doi:10.1016/j.uclim.2017.01.006

Messer, H., Zinevich, A., \& Alpert, P. (2006). Environmental monitoring by wireless communication networks. Science, 312(5774), 713-713.

Moltchanov, S., Levy, I., Etzion, Y., Lerner, U., Broday, D. M., \& Fishbain, B. (2015). On the feasibility of measuring urban air pollution by wireless distributed sensor networks. Elsevier. doi:10.1016/j.scitotenv.2014.09.059 
Morgan Stanley. (2018, December 02). Flying cars: Investment implications of autonomous urban air mobility. Retrieved from https://www.morganstanley.com/ideas/autonomous-aircraft

Morita, N., Hayakawa, M., \& Takao, N. (2018). Disaster prevention system utilizing social media information. Fujitsu Scientific and Technical Journal, 54, 54-58.

Muller, C. L., Chapman, L., Johnston, S., Kidd, C., Illingworth, S., Foody, G., . . . Leigh, R. R. (2015). Crowdsourcing for climate and atmospheric sciences: Current status and future potential. International Journal of Climatology, 35(11), 3185-3203. https://doi.org/10.1002/joc.4210

Napoly, A., Grassmann, T., Meier, F., \& Fenner, D. (2018). Development and application of a statistically-based quality control for crowdsourced air temperature data. Frontiers in Earth Science. https://doi.org/10.3389/feart.2018.00118

NCEP Central Operations. (2018, July 25). Madis. https://madis.ncep.noaa.gov

Niforatos, E., Vourvopoulos, A., \& Langheinrich, M. (2017). Understanding the potential of human-machine crowdsourcing for weather data. International Journal of Human - Computer Studies, 102, 54-68. doi:10.1016/j.ijhcs.2016.10.002

Oke, T. R. (1976). The distinction between canopy and boundary-layer urban heat islands. Atmosphere, 14(4), 268-277. doi:10.1080/00046973.1976.9648422

Osborne, M. J., \& Rubinstein, A. (1994). Course in game theory. MIT Press.

Ota, K., Dong, M., Gui, J., \& Liu, A. (2018, March). Quoin: Incentive mechanisms for crowd sensing networks. IEEE Network, 32(2), 114-119.

Overeem, A., Leijnse, H., \& Uijlenhoet, R. (2011). Measuring urban rainfall using microwave links from commercial cellular communication networks. Water Resources Research. doi:10.1029/2010WR010350

Overeem, A., Leijnse, H., \& Uijlenhoet, R. (2013a). Country-wide rainfall maps from cellular communication networks. Proceedings of the National Academy of Sciences of the United States of America. doi:10.1073/pnas.1217961110

Overeem, A., Robinson, J. C. R., Leijnse, H., Steeneveld, G. J., Horn, B. K. P., \& Uijlenhoet, R. (2013b). Crowdsourcing urban air temperatures from smartphone battery temperatures. Geophysical Research Letters, 40(15), 4081-4085. doi:10.1002/grl.50786

Pan, Z., Yu, H., Miao, C., \& Leung, C. (2017). Crowdsensing air quality with camera-enabled mobile devices. Proceedings of the Twenty-Ninth AAAI Conference on Innovative Applications, San Francisco, CA, AAAI, 47284733. 
Porsche Consulting. (2018). The future of vertical mobility: Sizing the market for passenger, inspection, and goods \& services until 2035. Retrieved from https://fedotov.co/wp-content/uploads/2018/03/Future-of-VerticalMobility.pdf

Price C., Maor R., \& Shachaf H. (2018). Using smartphones for monitoring atmospheric tides. Journal of Atmospheric and Solar-Terrestrial Physics, $174,1-4$.

Rabiei, E., Haberlandt, U., Sester, M., \& Fitzner, D. (2013). Rainfall estimation using moving cars as rain gauges - laboratory experiments. Hydrology and Earth Systems Sciences, 17, 4701-4712. doi:10.5194/hess-17-4701-2013

Rana, R., Chou, C., Kanhere, S., Bulusu, N., \& Hu, W. (2010). Ear-phone: An end-to-end participatory urban noise mapping system. Paper presented at the 105-116. Proceedings of the 9th ACM/IEEE International Conference on Information Processing in Sensor Networks, April 2010. https://doi.org/10.1145/1791212.1791226

Ronda, R. J., Steeneveld, G. J., Heusinkveld, B. G., Attema, J. J., \& Holtslag, A. A. M. (2017). Urban finescale forecasting reveals weather conditions with unprecedented detail. Bulletin of the American Meteorological Society, 98(12), 2675-2688. doi:10.1175/BAMS-D-16-0297.1

Ross, D. (2019, Winter). Game theory. The Stanford Encyclopedia of Philosophy, E. N. Zalta (Ed.). Retrieved from https://plato.stanford.edu/archives/ win2019/entries/game-theory

Rossi, C., Acerbo, F. S., Ylinen, K., Juga, I., Nurmi, P., Bosca, A., . . Alikadic, A. (2018). Early detection and information extraction for weather-induced floods using social media streams. International Journal of Disaster Risk Reduction, 30, 145-157. doi:10.1016/j.ijdrr.2018.03.002

Schweizer, I., Meurisch, C., Gedeon, J., Bärtl, R., \& Mühlhäuser, M. (2012). Noisemap: Multi-tier incentive mechanisms for participative urban sensing. Proceedings of the Third International Workshop on Sensing Applications on Mobile Phones, November 2012. https://doi.org/10.1145/2389148.2389157

Sendik, O., \& Messer, H. (2015). A new approach to precipitation monitoring: A critical survey of existing technologies and challenges IEEE. doi:10.1109/MSP.2014.2309705

Smith, I. (2012). The Internet of things. Halifax (UK): CASAGRAS2. ISBN 9780-9553707-9-3.

Snik, F., Rietjens, J. H. H., Apituley, A., Volten, H., Mijling, B., Di Noia, A., . . Keller, C. U. (2014). Mapping atmospheric aerosols with a citizen science network of smartphone spectropolarimeters. Geophysical Research Letters, 41(20), 2014, 41(20), 7351-7358. doi:10.1002/2014GL061462 
Sobash, R. A., \& Stensrud, D. J. (2015). Assimilating surface mesonet observations with the EnKF to improve ensemble forecasts of convection initiation on 29 May 2012. Monthly Weather Review, 143(9), 3700-3725.

Sosko, S., \& Dalyot, S. (2017). Crowdsourcing user-generated mobile sensor weather data for densifying static geosensor networks. ISPRS International Journal of Geo-Information, 6(3), 61. doi:10.3390/ijgi6030061

Steeneveld, G. J., Koopmans, S., Heusinkveld, B. G., Hove, V., B, \& Holtslag, A. A. M. (2011). Quantifying urban heat island effects and human comfort for cities of variable size and urban morphology in the Netherlands. Journal of Geophysical Research: Atmospheres, 116(D20), 1FF-n/a. doi:10.1029/2011JD015988

Strangeways, I. (2018). Phenology: Plants and animals as meteorological sensors. Weather, 73(3), 86-89. doi:10.1002/wea.2993

Sun, J. (2013). Incentive mechanisms for mobile crowd sensing: Current states and challenges of work. arXiv:1310.8364

Troemel, S., Ziegert, M., \& Ryzhkov, A. V., Chwala, C., \& Simmer, C. (2014). Using microwave backhaul links to optimize the performance of algorithms for rainfall estimation and attenuation correction. Journal of Atmospheric and Oceanic Technology, 31(8) 1748-1760. doi:10.1175/JTECH-D-14-00016.1

Trub, R., Moser, D., Schafer, M., Pinheiro, R., \& Lenders, V. (2018). Monitoring meteorological parameters with crowdsourced air traffic control data. Proceedings of the 17th ACM/IEEE International Conference on Information Processing in Sensor Networks April 2018, 25-36. https://doi.org/10.1109/IPSN.2018.00010

Tsujimori, T., Thepvilojanapong, N., Ohta, Y., Wang, H., Zhao, Y., \& Tobe, Y. (2013). SenseUtil: Utility vs. incentives in participatory sensing. Retrieved from http://www.greenorbs.org/events/pdf/hpb201303senseutil.pdf

Uber. (2016, October 27). Uber elevate, fast-forwarding to a future of on-demand urban air transportation. Retrieved from https://www.uber.com/ elevate.pdf

Uijlenhoet, R., Overeem, A., \& Leijnse, H. (2018). Opportunistic remote sensing of rainfall using microwave links from cellular communication networks. John Wiley \& Sons. doi:10.1002/wat2.1289

United Nations, Department of Economic and Social Affairs, Population Division. (2019). World urbanization prospects: The 2018 revision (ST/ESA/SER.A/420). New York: United Nations.

Unmanned Airspace. (2018a, October 11). There are now 50 cities around the world pioneering urban air mobility programmes. Retrieved from 
https://www.unmannedairspace.info/urban-air-mobility/now-50-citiesaround-world-pioneering-urban-air-mobility-programmes

Unmanned Airspace. (2018b, December 10). Urban air mobility takes off in 64 towns and cities worldwide. https://www.unmannedairspace.info/urbanair-mobility/urban-air-mobility-takes-off-63-towns-cities-worldwide

Vos, D., Lotte, Leijnse, H., Overeem, A., \& Uijlenhoet, R. (2017). The potential of urban rainfall monitoring with crowdsourced automatic weather stations in Amsterdam. Hydrology and Earth System Sciences, 21(2), 765-777. doi:10.5194/hess-21-765-2017

Vos, L. W., Raupach, T. H., Leijnse, H., Overeem, A., Berne, A., \& Uijlenhoet, R. (2018). High-resolution simulation study exploring the potential of radars, crowdsourced personal weather stations, and commercial microwave links to monitor Small-Scale urban rainfall. Water Resources Research, 54(12), 10, 293-10, 312. doi:10.1029/2018WR023393

Warren, E. L., Young, D. T., Chapman, L., Muller, C., Grimmond, C. S. B., \& Cai, X. (2016). The Birmingham urban climate laboratory-A high density, urban meteorological dataset, from 2012-2014. Scientific Data, 3(1), 160038. doi:10.1038/sdata.2016.38

Wolters, D., \& Brandsma, T. (2012). Estimating the urban heat island in residential areas in the Netherlands using observations by weather amateurs. Journal of Applied Meteorology and Climatology, 51(4), 711721. doi:10.1175/JAMC-D-11-0135.1

Wong, C. J., MatJafri, M. Z., Abdullah, K., \& Lim, H. S. (2009). Temporal and spatial air quality monitoring using internet surveillance camera and ALOS satellite image. Paper presented at the 2009 IEEE Aerospace conference, 1-7. doi:10.1109/AERO.2009.4839318

Wong, C. J., MatJafri, M. Z., Abdullah, K., Lim, H. S., \& Low, K. L. (2007). Temporal air quality monitoring using surveillance camera. Paper presented at the 2007 IEEE International Geoscience and Remote Sensing Symposium, 2864-2868. doi:10.1109/IGARSS.2007.4423441

$\mathrm{Xu}$, E. (2020, January 15). The future of transportation: White paper on urban air mobility systems. EHang Holdings Limited. Retrieved from https://www.ehang.com/app/en/EHang\%20White\%20Paper\%20on\%20Ur ban\%20Air\%20Mobility\%20Systems.pdf

Yang, D., Xue, G., Fang, X., \& Tang, J. (2012). Crowdsourcing to smartphones: Incentive mechanism design for mobile phone sensing. Proceedings of the ACM International Conference on Mobile Computing and Networking, 2012, 173-184.

Zhang, X., Yang, Z., Sun, W., Liu, Y., Tang, S., Xing, K. \& Mao, X. (2016). Incentives for mobile crowd sensing: A survey. IEEE Communications Surveys Tutorials, 18(1), 54-67. 
Zhu, Y., Zhang, S., Li, Y., Lu, H., Shi, K., \& Niu, Z. (2019). Social weather: A review of crowdsourcing-assisted meteorological knowledge services through social cyberspace. Geoscience Data Journal. https://doi.org/10.1002/gdj3.85

Zinevich, A., Alpert, P., \& Messer, H. (2008). Estimation of rainfall fields using commercial microwave communication networks of variable density. Advances in Water Resources, 3(11), 1470-1480. doi:10.1016/j.advwatres.2008.03.003

Zinevich, A., Messer, H., \& Alpert, P. (2010). Prediction of rainfall intensity measurement errors using commercial microwave communication links. Katlenburg-Lindau: Copernicus GmbH. doi:10.5194/amt-3-1385-2010 